\title{
¿Qué se requiere realmente para contar con compras gubernamentales más eficien- tes? ¿Basta con modificaciones normativas? Una mirada a los procesos de selección sujetos a la legislación de contrataciones del Estado
}

\section{What is actually required to have government procurement more efficient? Is it enough to change policy? A look at the selection process subject to state procurement laws}

\author{
SOFÍA PRUDENCIO GAMIO”
}

Resumen: Mediante la formulación de distintas preguntas, se analiza en qué consiste un proceso de selección y cuáles son sus principales características. El artículo se enfoca en explicar que, más allá de ser un conjunto de procedimiento, el proceso de selección implica tener un aparato estatal correctamente capacitado para dar una respuesta rápida y eficiente. Asimismo, se explica la necesidad de encontrar un equilibrio entre el marco normativo que controle las desviaciones de poder y el empoderamiento de los funcionarios encargados de las compras gubernamentales.

Palabras clave: Proceso de selección - compras gubernamentales contrataciones del Estado - bases, consultas y observaciones - presentación de propuestas

Abstract: Through the development of various questions it is discussed what a selection process is and what its main features are. The article focuses on explaining that beyond being a set of procedures, the selection process involves having a properly trained state apparatus to give respond quickly and efficiently. It also explains the need to strike a balance between the regulatory framework to control the abuses of power and empowerment of the civil servants responsible for government procurement.

Key words: Selection process - procurement - contracting State - databases queries and comments - submission of proposals

* Es abogada especialista en contrataciones públicas y directora de Supervisión, Fiscalización y Estudios del Organismo Supervisor de Contrataciones del Estado. Las opiniones vertidas en este artículo son responsabilidad exclusiva de la autora y no reflejan necesariamente la posición institucional del Organismo Supervisor de Contrataciones del Estado. 


\section{INTRODUCCIÓN}

Si bien existen varios casos de compras gubernamentales exitosas, ejecutadas bajo los principios y normas vigentes, no resulta novedad que los actuales procedimientos presenten debilidades que deben ser corregidas, y hacia ello se apunta vía el desarrollo de las plataformas transaccionales del Sistema Electrónico de Contrataciones del Estado (seace) y el impulso de modalidades como el convenio marco. Sin embargo, en tanto dicha optimización se concrete, iqué les espera a los procesos de selección?

Atendiendo a tal interrogante, el objetivo de este artículo es llamar la atención sobre el rol de aquellos funcionarios y colaboradores del Estado encargados de articular los procesos de selección. Así, a partir de la identificación de algunas prácticas adoptadas por entidades convocantes de procesos de selección, me permito compartir esta reflexión sobre el principal ingrediente para contar con compras gubernamentales más eficientes.

\section{I. ¿QUÉ ES UN PROCESO DE SELECCIÓN?}

El anexo de Definiciones del Reglamento de la Ley de Contrataciones del Estado', en adelante el reglamento, conceptúa el proceso de selección como un procedimiento administrativo especial conformado por un conjunto de actos administrativos, de administración o de hechos administrativos que tienen por objeto la selección de la persona natural o jurídica con la cual las entidades del Estado van a celebrar un contrato para la contratación de bienes, servicios u obras.

Por su parte, la doctrina define un proceso de selección como "el procedimiento administrativo de preparación de la voluntad contractual, por el que un ente público en ejercicio de la función administrativa invita a los interesados para que, sujetándose a las bases fijadas en el pliego de condiciones, formulen propuestas de entre las cuales seleccionará y aceptará las más convenientes"’.

Estos procedimientos, conforme con la Ley de Contrataciones del Estado $^{3}$, en adelante la ley, se traducen en cuatro procesos de selección, comúnmente llamados "tradicionales" para diferenciarlos de aquellos sujetos a las nuevas modalidades de contratación, como son la subasta inversa y el convenio marco ${ }^{4}$.

1 Aprobado mediante decreto supremo 184-2008-EF.

2 Dromi, Roberto. Licitación pública. Buenos Aires: Ciudad Argentina, 2006, p. 172.

3 Aprobada por decreto legislativo 1017.

4 Artículo 15 de la Ley de Contrataciones del Estado: "Los procesos de selección son: Licitación Pública, concurso público, adjudicación directa y adjudicación de menor cuantía, los cuales se podrán realizar de manera corporativa o sujetos a las modalidades de selección de subasta inversa o convenio marco, de acuerdo a lo que defina el Reglamento". 
Ahora bien, los procesos de selección se sustentan en determinados principios. Los más relevantes, a efectos de este artículo, son los principios de transparencia, libre concurrencia y competencia, publicidad, eficiencia, moralidad, y trato justo e igualitario.

Sobre el particular, es doctrinariamente reconocido que los procesos de selección se sustentan en el principio de libre concurrencia y competencia, pues "tiene que haber libertad, entendida como la posibilidad de participar en el procedimiento de selección del contratista en forma libre de coacción, tanto en lo referido al ingreso por propia iniciativa a la licitación, como durante el desenvolvimiento del proceso"s.

Asimismo, tal libertad debe estar acompañada de la transparencia, pues "es un arma eficaz en la lucha contra la corrupción y los fraudes de todo tipo respecto de los fondos públicos".

De esta manera, los procesos de selección están concebidos para que las entidades del Estado obtengan las mejores condiciones para la satisfacción de sus necesidades (calidad, cantidad, precio, plazos) en salvaguarda del interés común, lo que a su vez impacta en la economía, dado el rol consumidor del Estado. No obstante, los principios también destacan la necesidad de rapidez en el desarrollo de los procedimientos, así como "la necesidad de evitar toda sospecha de inmoralidad o corrupción en la contratación misma: la debida publicidad del procedimiento y la implementación de mecanismos idóneos de control garantizan la vigencia y el respeto de los principios de igualdad, concurrencia y transparencia de la contratación"?.

Dicho así, la razonabilidad debería ser más que suficiente para llevar a cabo compras gubernamentales exitosas; sin embargo, la realidad muestra algo distinto. En efecto, el Estado peruano se ha provisto de procedimientos reglados, etapas preclusivas y plazos perentorios, todo lo cual debe ser articulado por los funcionarios de las entidades.

\section{LA REALIDAD}

Según se aprecia en la información registrada en el portal del Organismo Supervisor de Contrataciones del Estado, durante el ejercicio de 2010 se realizaron 83241 procesos de selección denominados "tradicionales", entre licitaciones públicas, concursos públicos, adjudicaciones directas públicas y selectivas, y adjudicaciones de menor cuantía, por un monto

5 Dromi, Roberto. Licitación pública. Ob. cit., p. 46.

6 Moreno Molina, José Antonio. La contratación pública en los países de la Comunidad Andina. Un estudio comparado con el Derecho de la Unión Europea. Lima: Secretaría General de la Comunidad Andina, Proyecto de Cooperación UE-CAN, 2007, p. 80.

7 Farrando, Ismael. Contratos Administrativos. Buenos Aires: Abeledo - Perrot, 2002, p. 372.

8 Reporte anual de contrataciones públicas 2010. Resultados preliminares. <http://www.osce.gob.pe>. Sección "Publicaciones".

¿QUÉ SE REQUIERE

REALMENTE

PARA CONTAR

CON COMPRAS

GUBERNAMENTALES MÁS EFICIENTES?

WHAT IS ACTUALLY REQUIREDTO HAVE GOVERNMENT PROCUREMENT MORE EFFICIENT? 
total de S/. 15343 millones. Lo expuesto revela que el Estado es uno de los mayores consumidores del mercado, por lo que no ha de extrañar que concentre la atención de más de 120 mil proveedores a nivel nacional.

Ahora bien, la realización de estos procesos de selección requiere de actos preparatorios previos cuyos principales productos, a los efectos del consecuente proceso, resultan ser las bases o pliegos de condiciones. Una vez aprobadas estas bases, la entidad se encuentra lista para dar inicio al proceso de selección de que se trate, el mismo que, como he señalado, tiene por objetivo la selección de la mejor oferta. En general, los procesos comprenden las siguientes etapas: convocatoria, registro de participantes, formulación y absolución de consultas y observaciones a las bases, integración de bases, presentación de propuestas, evaluación y otorgamiento de la buena pro.

Dicho ello, veamos qué muestra la información de acceso público registrada en el seace en relación con el rol que desempeñan aquellos llamados a dar vida a los procesos de selección.

\section{II.1. Bases}

El reglamento define las bases como aquellos documentos que contienen el conjunto de reglas formuladas por la entidad convocante y que especifican el objeto del proceso, las condiciones a seguir en la preparación y ejecución del contrato, y los derechos y obligaciones de los participantes, postores y el futuro contratista.

Esta definición es compartida a nivel internacional, al considerarse las bases como reglamentos a cuyas disposiciones deben sujetarse tanto el Estado como los proveedores interesados. De esta manera, las bases se convierten en el parámetro para medir la actuación de ambos protagonistas:

[...] se trata de normas contenidas en un reglamento administrativo que tienden a homogeneizar los criterios de evaluación, permitiendo así tanto el control de legalidad por parte de la Administración, como el resguardo de los propios derechos de los participantes, que también requieren de datos objetivos a esos efectos. Se trata, en definitiva, de garantizar los principios de publicidad — conocimiento de las razones tenidas en cuenta por la Administración—, competencia — pujar conforme a los mismos criterios de selección — e igualdad — trato a todos los concursantes oferentes sin discriminación ni preferencias subjetivas-, principios estos esenciales a todo procedimiento administrativo de selección, y emanados de las garantías del debido proceso y de igualdad $[\ldots]^{9}$.

9 Casagne, Juan Carlos. El contrato administrativo. Segunda edición. Buenos Aires: Abeledo - Perrot, 2005, p. 98. 
Entonces, formalmente no quedan dudas sobre la importancia de estos documentos para la consecución de los procesos de selección. Es claro para la normativa peruana y para la legislación comparada que estos documentos rigen los procesos, por lo que deben ser lo suficientemente claros, concretos y objetivos, así como debidamente publicitados.

Ahora bien, los invito a pensar en alguna necesidad particular: por ejemplo, la necesidad de adquirir un vehículo para un negocio. Al tener claros los objetivos del negocio y los recursos con que se cuenta - producto del ahorro de varios años o de algún préstamo familiar o bancario-, la selección de nuestro proveedor no puede tomarse a la ligera. Así, un empresario privado pensaría que, si la compra del vehículo no resulta — sea porque la entrega se retrasa, el bien resulta defectuoso o finalmente no puede ser utilizado por habernos equivocado en cuanto a las especificaciones-, el negocio no tendrá éxito. Por ello coincidirán en que las bases que planteemos para la selección de nuestro proveedor deben fomentar una competencia célere pero transparente, sin ser elaboradas con negligencia y, mucho menos, con dolo.

Desafortunadamente, pese a que en las compras gubernamentales el "negocio" es el cumplimiento de los objetivos institucionales — los que por su naturaleza impactan en la sociedad-, puede identificarse en algunas entidades del Estado cierta vocación por arriesgar graciosamente los recursos que respaldan tales compras al dirigirlas a determinados proveedores por intereses distintos de la búsqueda de la mejor oferta.

En relación con lo expuesto, este es un extracto de las bases de una Adjudicación Directa Selectiva registrada en el seace, convocada para la contratación del consultor que elabore un estudio definitivo para un proyecto de infraestructura de riego:

Ojo Darcy en esta parte de las bases es fundamental que lo veas de acuerdo a tu perfil obviamente va la consulta que si tu como agrónomo puedes y estás facultado para la formulación de proyectos de este tipo de manera que si es así enriquece los cuadros pero Darcy tú te puedes finalmente presentar como empresa consultora y puedes proponer a los ingenieros civiles economistas y agrícolas que sí están involucrados en este tipo de trabajos. Necesitamos Darcy colgar mañana el proceso [...] dale una chequeadita [a] la parte de los términos de referencia tal vez algo falte y debe de concordar con el expediente que has preparado [sic].

Como se advierte, esta nota aparentemente olvidada en el texto de las bases revelaría acuerdos previos con un proveedor en particular, lo que trasgrede los principios anteriormente comentados. Lógicamente, y según se aprecia de la ficha del proceso publicada en el seace, la entidad convocante declaró la nulidad del proceso y dispuso el inicio de 
las acciones conducentes al deslinde de responsabilidades; no obstante, cabría preguntarle a los potenciales proveedores si en lo sucesivo podrían confiar en la actuación de la entidad. Al evidenciarse el direccionamiento hacia un determinado proveedor, isería posible pensar que se buscaba una compra exitosa? ¿Dónde quedó la observancia de los principios de libre competencia y de moralidad?

\title{
II.2. Convocatoria
}

Una vez que ha quedado claro que las bases son las reglas del proceso, resulta lógico que estas deban ser conocidas para poder surtir efectos. Esta difusión se denomina en doctrina "llamado" y resulta fundamental pues "a través de él nace jurídicamente el procedimiento administrativo en su faz externa a la administración y pone en vigencia toda la normativa que $[. .$.$] necesitaba de la publicación para su eficacia" { }^{10}$.

Ahora bien, el artículo 51 del reglamento establece que las bases deben ser publicadas en el seace con ocasión de la convocatoria, bajo sanción de nulidad.

Podría resultar curioso que una situación lógica como la indicada deba ser protegida con la sanción de nulidad; sin embargo, casos como el que veremos a continuación parecen sustentar la necesidad de precisar el grave efecto de no registrar las bases en la misma oportunidad de la convocatoria:

\section{REF: OF.N ${ }^{\circ}$ 1096-10-ABAST-OCEP-UNP}

(H.T. N ${ }^{\circ}$ 000385-5403-10-5).

\begin{abstract}
APROB. DE BASES ADMIN. ADS N 0031-2010-UNP"ADQUISICIÓN DE MATERIAL DE ENSEÑANZA PARA LAS DIFERENTES UNIDADES OPERATIVAS DE LA UNP"
\end{abstract}

Sin otro particular, me despido de usted.

Atentamente

El documento arriba reseñado fue el único registrado en el seace, en el campo reservado para las bases. En esta situación, icómo podrían los proveedores o los usuarios finales de la compra, enterarse de las condiciones exigidas por la entidad? 
Otra variante es que el archivo que contiene las bases sea registrado cifrado, o que las bases sean publicadas en forma incompleta. En este escenario, los proveedores se ven obligados a recurrir a la entidad convocante para solicitar la documentación faltante. Esta situación no solo encarece la participación de postores, sino que limitaría la competencia.

En efecto, si bien formalmente se registró información, esta no puede ser conocida en su integridad o no se corresponde con el objeto contractual, lo que trasgrede el principio de publicidad, consagrado no solo en la normativa nacional sino también por la legislación comparada:

El régimen de publicidad constituye el verdadero pilar sobre el que se asienta el sistema de contratación pública establecido por la normativa comunitaria europea. Así lo ha señalado el Tribunal de Justicia de las Comunidades Europeas, al resaltar que una publicidad adecuada de los anuncios de licitación ofrece a todos los contratistas interesados de la Comunidad la posibilidad de ser informados sobre la licitación y, en su caso, de participar en ella (Sentencia de 18 de marzo de 1992, Comisión contra España, Asunto C-24/91, Rec. 1992, p. I-1989, FJ. 2). Tanto el principio de concurrencia como el de igualdad necesitan como requisito imprescindible la existencia de una adecuada publicidad de la convocatoria ${ }^{11}$.

En cuanto a los efectos de tal comportamiento de la entidad convocante, el costo resultaría bastante alto pues es tal la gravedad del vicio que solamente podría ser corregido vía la declaración de nulidad:

[...] una defectuosa publicidad puede estar atacando otros elementos del acto, ya que la misma puede haber tenido por objetivo el excluir a algún tipo de oferentes (de alguna localidad) y ello afectaría a otros elementos del acto del llamado como su causa o finalidad [...] desde la publicación del llamado pueden alterarse los principios mismos de la licitación como procedimiento. Puede quedar afectada la generalidad o libre concurrencia si la publicación se realizó informando defectuosamente el producto que se requiere o el tipo de contrato que en realidad se persigue celebrar; puede alterarse el principio de igualdad si la publicación se realiza con ocultamiento de información en su contenido que luego resulta ser conocida por algún "allegado" al funcionario; finalmente, también se puede afectar el principio de publicidad [...] La carencia de alguno de los requisitos que hemos señalado o su defectuoso cumplimiento pueden dar lugar a la existencia de vicios en el llamado. Estos vicios deben ser corregidos a instancias de la misma Administración o a pedido de los interesados $[\ldots]^{12}$.

¿QUÉ SE REQUIERE REALMENTE

PARA CONTAR

CON COMPRAS

GUBERNAMENTALES MÁS EFICIENTES?

WHAT IS ACTUALLY REQUIRED TO HAVE GOVERNMENT PROCUREMENT MORE EFFICIENT? 
Cabe preguntarse entonces si es la normativa la causante de la situación descrita y quién asume el costo de tales errores.

\section{II.3. Registro de participantes}

"El tesorero se encuentra de viaje por motivos de salud de su menor hijo, su ausencia es hasta el 10 de febrero del año en curso [...] el secretario no está autorizado para la venta de Bases ni tampoco conoce del proceso de selección [sic]". Declaraciones de este tipo podrían resultar anecdóticas si no fuera porque en los últimos tiempos se han convertido en constantes que los proveedores deben enfrentar cuando se acercan a las sedes institucionales de algunas entidades para registrarse como participantes.

En efecto, según el anexo de Definiciones del Reglamento, "participante" es el proveedor que puede intervenir en un proceso de selección por haberse registrado conforme a las reglas establecidas en las bases. Por su parte, el artículo 52 de la referida norma prescribe que la persona que se registra como participante se adhiere al proceso de selección en el estado en que este se encuentre. Así, se desprende de la normativa que el proveedor sería el responsable de registrarse y que, de no hacerlo adecuadamente o de hacerlo tarde, debe asumir las consecuencias.

No obstante, al tratarse de procesos de selección distintos de las adjudicaciones de menor cuantía electrónicas, el registro no depende exclusivamente del proveedor, sino que requiere de la intervención de la entidad convocante, sea para el cobro del derecho de participación y la recepción del formato de datos del proveedor, sea para la entrega de un ejemplar de las bases o del expediente técnico, en caso corresponda.

En ese sentido, si bien la normativa atribuye al proveedor la responsabilidad de su registro como participante, queda claro que la consecución de tal acto indispensable para poder intervenir no depende exclusivamente de él. Ahora bien, si contar con mayor número de proveedores aseguraría en cierta medida resultados más eficientes, ¿por qué obstaculizar o, inclusive, impedir el registro de determinados participantes?

Al respecto, es importante considerar que, para la Comisión de Alto Nivel Anticorrupción, "cuando el usuario no ve otra opción ante un trámite o proceso que desea realizar ante el Estado y más aún si dicho proceso se vuelve ineficiente, posiblemente adrede, termina cayendo ante la opción de ofrecer coimas a los funcionarios para hacer que el proceso funcione a su favor"13 (el subrayado es agregado). 
En esa medida, el registro de participantes se presenta como una etapa vulnerable a los actos de corrupción. Ello por cuanto, como se ha señalado, no depende exclusivamente del proveedor, sino que amerita de una actuación de la entidad convocante. Por tal razón, se ha convertido en práctica usual que los proveedores se acompañen de notarios, jueces o miembros de las fuerzas policiales a fin de que quede constancia del desconocimiento o de la resistencia de los funcionarios ante el registro, todo lo cual, también, encarece la participación en los procesos de selección al afectar la mayor participación.

Sin embargo, esta vulnerabilidad no es exclusiva del registro de participantes.

\section{II.4. Consultas y observaciones}

De acuerdo con la normativa vigente, es responsabilidad del Comité Especial absolver de manera fundamentada las consultas y observaciones que, respecto de las bases - en el entendido de que hayan sido bien registradas-, hayan sido formuladas por los proveedores — que hayan logrado inscribirse como participantes-.

Sin embargo, basta con revisar algunas fichas de procesos registradas en el seace, para encontrar casos en los cuales la entidad convocante, distorsionando la prerrogativa concedida, absuelve observaciones de la siguiente manera:

Al momento de integrar las bases se rectificarán inconsistencias. Si las hay.

1. Al momento de integrar las Bases se rectificarán inconsistencias. Si las hay.

2. Se tendrá en cuenta.

3. Ídem.

4. Se le da la razón al consultor.

5. Se tendrá en cuenta al momento de integrar las Bases.

Como se aprecia, tales respuestas no solo no son fundamentadas, sino que no indican en qué forma se modificarán las bases. $\mathrm{Al}$ respecto, basta con recordar los principios que rigen los procesos de selección para concluir que la ausencia de transparencia revelada por la conducta reseñada no contribuye en lo más mínimo a fomentar la mayor participación.

Adicionalmente, lo expuesto constituye un vicio que afecta la validez de los actos posteriores del proceso, lo que en sendas oportunidades ha sido reconocido por el Tribunal de Contrataciones del Estado y da lugar a la nulidad del proceso: 
En esta línea, siendo que [...] las entidades se encuentran en la obligación de absolver las consultas y/u observaciones presentadas oportunamente en el proceso de selección y, asimismo, que en el presente caso se ha evidenciado que el comité especial omitió dicha obligación, es posible concluir que se ha configurado la causal de nulidad referida a la contravención de las normas contenidas en los artículos 45으, 97으 y $115^{\circ}$ del Reglamento, de conformidad con lo establecido en el artículo $57^{\circ}$ de la Ley $[\ldots]^{14}$.

Ahora bien, si nos situamos en el momento en que los proveedores toman conocimiento de la referida absolución de observaciones, ante el incumplimiento de la normativa no les quedaría más opción que solicitar la elevación de observaciones. Pero iqué sucede cuando es la propia entidad convocante la que debe pronunciarse al respecto?

En efecto, de conformidad con lo establecido en el artículo 28 de la ley, si el valor referencial del proceso es inferior a 300 Unidades Impositivas Tributarias (UIT), las observaciones serán absueltas por el titular de la entidad convocante en última instancia.

Así, en el caso comentado precedentemente, se registró el siguiente pronunciamiento:

De mi especial consideración,

Con mis cordiales saludos me dirijo a Usted y su representada a fin de comunicarle que la Municipalidad que presido ha recibido su documento y ha visto por conveniente darle razón en parte; acción que podrá constatar en las Bases Integradas.

Renuevo a Usted las muestras de mi más distinguida consideración y deferencia personal [sic].

¿Acaso no es el artículo 58 del reglamento el que prescribe que el pronunciamiento — que es inimpugnable — debe ser motivado y expresado de manera objetiva y clara, y que deben absolverse en él las observaciones y, de ser el caso, pronunciarse de oficio sobre cualquier aspecto de las bases que contravenga la normativa de contrataciones del Estado?

De otro lado, si se atiende a los objetivos que se persiguen con los procesos de selección, ino debería interesarle a la entidad convocante, inclusive más que a los propios proveedores, el cabal conocimiento de las bases, pues de su adecuado análisis depende la presentación de ofertas serias y coherentes?

14 Resolución 1180/2007.TC-S4 emitida por el Tribunal de Contrataciones del Estado. <http://www. osce.gob.pe>. Si bien el proceso impugnado se llevó a cabo bajo la vigencia del decreto supremo 083-2004-EF, cabe considerar que las disposiciones infringidas también se encuentran consagradas en la normativa vigente. 
Sobre el particular, cabe hacer mención al principio de legalidad, consagrado en la ley 27444, Ley del Procedimiento Administrativo General. Según este principio, las autoridades de la administración deben actuar, según la Constitución, la ley y el Derecho, dentro de las facultades que les estén atribuidas y de acuerdo con los fines para los que les fueron conferidas. Ello supone que el Estado actúe bajo parámetros claros, en salvaguarda de los derechos de los administrados, pues "la concepción según la cual el Estado podía hacer lo que no le estuviera prohibido, abría un ilimitado campo de acción a su discrecionalidad que le permitía aparecer en todos los espacios francos o silenciados por la ley expresa, con lo cual se toleraba la existencia de actos administrativos sin parámetro de contraste que permita evaluar su legitimidad"15.

Sin embargo, en el ejemplo mostrado no se advierte ejercicio alguno de la facultad conferida por la normativa, lo que, nuevamente, afecta la transparencia del proceso de selección y pone en riesgo los recursos que respaldan las compras. En esta línea, pese a la concepción generalizada de que el marco normativo debe ser flexibilizado a fin de que las compras gubernamentales sean más céleres, debería evaluarse primero si existen en la generalidad de entidades la aptitud y actitud suficientes para asumir tal responsabilidad. Lo contrario podría suponer una mayor exposición a actos de corrupción pues "Al tener el funcionario público la seguridad de que el usuario no tiene otra opción y no existe otra instancia ante quien pueda acudir, la posibilidad de verse tentado a cometer un abuso de ese poder y cometer un acto corrupto es alta. Este fenómeno genera el síndrome del dictador que asume que puede tomar cualquier decisión y que está premunido de inmunidad"16.

\subsection{Presentación de propuestas}

Conforme se advierte del reglamento, el acto público de presentación de propuestas es formal. Tan es así que "si al momento de ser llamado el participante no se encuentra presente, se le tendrá por desistido".

Sobre el particular, es compartido por la doctrina que:

[...] el vencimiento del plazo es «fatal», no rigiendo aquí el principio de «informalismo a favor del administrado»; es decir que vencido el plazo, vencido está el derecho a participar en el procedimiento licitatorio, y no puede ser ampliado individualmente a ningún oferente por ninguna razón, aun cuando se alegare caso fortuito o fuerza mayor por parte de aquéllos. El cumplimiento estricto del plazo es uno de los principios rectores que aseguran el cumplimiento efectivo del principio de igualdad

¿QUÉ SE REQUIERE REALMENTE

PARA CONTAR

CON COMPRAS

GUBERNAMENTALES MÁS EFICIENTES?

WHAT IS ACTUALLY REQUIRED TO HAVE GOVERNMENT PROCUREMENT MORE EFFICIENT? 
de los oferentes. Las ofertas deben ser presentadas dentro del término que fija el licitante en el llamado a licitación, Cualquier presentación tardía debe ser rechazada porque pone en juego al principio de igualdad de los oferentes ${ }^{17}$.

En esa medida, tal cual el estado de nuestra legislación, ingresar diez minutos tarde al recinto donde se llevará a cabo el acto público daría lugar a que el participante que se registró primero no pueda presentar su oferta.

Dicho esto, y tal como sucede con el registro de participantes, los actos públicos no se encuentran libres de actuaciones que afectan los principios de contratación pública. Así, conforme a una ocurrencia policial solicitada por un proveedor a quien se le impidió el ingreso a la oficina donde debían presentarse las ofertas:

Se pudo constatar que la puerta del local de administración donde se estaba llevando a cabo el acto público de dicha licitación se encontraba cerrada $[\ldots]$ asimismo se encontró en la parte de afuera al señor Teniente Alcalde $[\ldots]$, al señor Teniente Gobernador [...] y al señor Tesorero [...] quienes manifestaron que la puerta de dicha oficina había sido cerrada a horas 08.50 am y que tampoco los habían dejado ingresar $[\ldots]$ y que en varias ocasiones han tocado la puerta para que los dejen ingresar con resultados negativos [sic].

Una variante de esta situación es la modificación de la fecha, horario o lugar donde se realizará el acto público de presentación de propuestas, sin la debida notificación previa a los participantes.

En cualquier caso, la conducta de los funcionarios encargados contraviene la normativa, y se advierte de ello la restricción - negligente o dolosa - de la mayor participación de postores, lo que desnaturaliza los procesos de selección.

$\mathrm{Al}$ respecto, un estudio de percepción sobre las vulnerabilidades del sistema de contrataciones públicas realizado en Argentina advierte que en la administración es común la creencia de que la transparencia y la reducción de los márgenes de discrecionalidad de un proceso de compras conducen a un trámite lento, conflictivo e ineficiente, por lo que se prefiere optar por la vía más corta y menos transparente. Sin embargo, conforme con este estudio:

[...] los funcionarios que tuvieron la experiencia de llevar adelante un proceso procurando procesos transparentes y respetando los principios aplicables entienden que en estos casos se producen efectos positivos que mejoran sustancialmente todos los aspectos de las contrataciones. 
Es decir, tuvieron la oportunidad de comprobar que el respeto del principio de legalidad y transparencia en las contrataciones públicas conduce a mejores resultados que los actuales ${ }^{18}$.

En tal sentido, la inobservancia de la normativa y los principios no constituye solución alguna; por el contrario, incrementa los riesgos de corrupción y desabastecimiento.

Por último, cabe referirme a un elemento de importancia en los actos públicos, como es la presencia del notario. Compete a este profesional, de acuerdo con el artículo 2 del decreto legislativo 1049, Ley del Notariado, dar fe de los actos y contratos que se celebren ante él. Sobre aquello, el artículo 30 de la ley y el artículo 64 de su reglamento consagran que la presencia del notario en los actos públicos es indispensable. No obstante, ¿qué sucede cuándo el notario público permite que se consigne en el acta respectiva situaciones que no se produjeron?

Conforme se advierte de la resolución 1122-2009-TC-S1, emitida con ocasión de la Adjudicación Directa Selectiva 001-2009-MPP, el notario interviniente en el acto dio fe de la devolución de una oferta. Sin embargo, tal devolución no se había realizado:

[...] fluye de los antecedentes reseñados que en el Acta levantada el 09 de marzo de 2009 [por] el Notario Público de la Provincia de Pacasmayo [...] que "[...] el comité especial acordó no admitir la propuesta presentada por la empresa postora se llamó al postor para que se le haga entrega de su propuesta, quien accedió a la misma firmado el cargo correspondiente".

No obstante, de acuerdo a lo aseverado por el Consorcio Inversiones \& Leansa, su representante se encontraba ausente; razón por la cual conservó su condición de postor. Esta afirmación resulta concordante con lo informado por la entidad en su comunicación del 02 de abril de 2009.

Conforme a lo expuesto, a pesar de que en el Acta del 09 de marzo de 2009 se consignó que el Consorcio Inversiones \& Leansa aceptó la devolución de su propuesta; este Colegiado estima que lo afirmado en el citado documento no refleja con exactitud los hechos acontecidos en el referido acto público, particularmente en lo que respecta a la participación del citado impugnante, no solo en virtud a lo informado por las partes intervinientes sino, más aún, debido a que la propia entidad ha remitido a este Colegiado el sobre de la propuesta económica del postor, el cual se encuentra debidamente lacrado con el sello del Notario Público presente en acto público.

18 Arcidiacono, Pilar, Gastón Rosemberg y Federico Arenoso. Contrataciones públicas vulnerables. Estudio de percepción sobre las vulnerabilidades del Sistema Nacional de Contrataciones Públicas. Primera edición. Buenos Aires: Fundación Poder Ciudadano / Área Transparencia y Anticorrupción / Programa Contrataciones Públicas Transparentes, 2006, p. 83.

¿QUÉ SE REQUIERE REALMENTE

PARA CONTAR

CON COMPRAS

GUBERNAMENTALES MÁS EFICIENTES?

WHAT IS ACTUALLY REQUIREDTO HAVE GOVERNMENT PROCUREMENT MORE EFFICIENT? 
En ese orden de ideas, este Colegiado estima que la existencia de eventuales errores u omisiones cometidas por parte del Notario Público [...], quien no habría supervisado que la información consignada en el instrumento público redactado refleje con exactitud las incidencias del acto ante él celebrado a efectos de otorgar fe pública y certeza plena de los hechos que presenció por razón de su función, la misma que le ha sido asignada por el Estado, de conformidad con lo previsto en el artículo 2 de la Ley del Notariado, no debe perjudicar el derecho que le asiste al Impugnante para contradecir los actos que considera lesivos a sus intereses; motivo por el cual se concluye que el recurso venido en grado resulta procedente, debiendo realizarse el análisis sobre el fondo del mismo, sin perjuicio de comunicar tales irregularidades a las instancias correspondientes a fin de que se determinen las responsabilidades consiguientes, de ser el caso [sic].

Se advierte, entonces, que en un proceso de selección pueden conjugarse varios elementos que no necesariamente contribuyen a la eficiencia de la compra.

Así, reiteramos la interrogante que dio inicio a este artículo: ¿qué les espera a los procesos de selección?

\section{CONCLUSIONES}

Las compras gubernamentales nunca serán solo un conjunto de procedimientos. En la medida en que deben ser articulados por personas, este se constituye en factor vital para el éxito de aquellas. Si se tiene eso en cuenta, y dada nuestra realidad, no es suficiente simplificar reglas o ajustar tiempos para obtener resultados eficientes en el corto plazo. Por el contrario, los ejemplos mostrados permiten concluir que los mayores esfuerzos deben apuntar a asegurar que el Estado cuente con funcionarios técnicamente idóneos, conscientes del reto que supone invertir adecuadamente los recursos estatales. Elaborar la estrategia de compra, preparar bases, conducir un proceso de selección, verificar el cumplimiento del contratista y generar retroalimentación para evitar los mismos defectos en la siguiente contratación constituyen actividades relevantes que exigen conocimientos y experiencia, así como interés. En este contexto, las compras gubernamentales no son producto de las normas sino de la gestión.

De otro lado, ninguno de los casos que hemos comentado podría justificarse en la rigurosidad de los procedimientos o en la búsqueda de celeridad; por el contrario, el apartamiento de los principios — con aparentes fines distintos de la eficiencia - eventualmente repercutirá en su continuidad — si es evidenciado a tiempo dando lugar a la nulidad—o 
en la pérdida de los recursos, por contratarse en condiciones perjudiciales para el Estado.

En adición, situaciones como las referidas contribuyen a que la percepción del Perú en relación con la corrupción sea alta. Así lo muestra el Índice de Percepción de Corrupción ${ }^{19}$, cuyo informe de 2009 sitúa al Perú con un índice de 3,7\%, en el puesto 75 de 180 países encuestados.

En este escenario, en adición a las acciones específicas en cada caso concreto, es necesario encontrar el equilibrio entre el marco normativo que controle las desviaciones de poder y el empoderamiento de los funcionarios encargados de las compras gubernamentales, quienes deben encontrarse capacitados y comprometidos con tan importante tarea. No lo considero imposible, pero cierto es que el camino es largo y sinuoso. El reto es, entonces, adoptar una visión integral de la eficiencia e interrelacionar las compras gubernamentales con los demás proyectos dirigidos a optimizar la especialización de la administración pública, rescatando el rol de la vocación en esta materia.

¿QUÉ SE REQUIERE REALMENTE

PARA CONTAR

CON COMPRAS

GUBERNAMENTALES MÁS EFICIENTES?

WHAT IS ACTUALLY REQUIRED TO HAVE

GOVERNMENT

PROCUREMENT

MORE EFFICIENT? 